\section{LES PRINCIPAUX}

\section{ACIERS DE CONSTRUCTION}

\author{
LEURS CARACTÉRISTIQUES -- LEURS EMPLOIS
}

Lorsqu'un constructeur veul élablir une machine, il cst souvent très embarrassé pour choisir l'acier qui conviendra le mieux à chacun des`organes rị'il vient d'éludier.

la lecture des catalogues des firmes mélallurgiques n’est pas toujours faite pour le guider d'une maniere bien nolle dians ses recherches. Anssi, en présence de l'incertitude dans laquelle il se trouve après celle consultation, il en revient le plus souvent aux nuances d'acier qu'il a pu expérimenter précédemment, renonçant à sc Jancer au hasard dans le dédale des acicrs spéciaux rqu'il no connaît pas.

Nonis avons déjà cu l'occasion d'insister sur ce point $\left(^{1}\right)$, mais nous croyons qu'il ne sera pas inutile d'y revenir ici.

Dans le but d'éclairer ses adhérents, la Chambre syndicalc des Constructeurs d'automobiles a fait un essai de standarisation des principaux aciers do construction. Elle a réuni, dans un lableau que nous reproduisons ci-après (tableau I), les principales caractéristiques chimiques et mécaniques des acicrs ainsi choisis. C'est ce lableau gui va servir de base à la pelite élude qui suit.

Cet essai de standarisation, qui peut paraître assez prétenlieux pour un profane, était d'autant plus justifié que les aciers spéciaux les plus employés se ramènent à un certain nombre de nuances reproduites d'une manière à peu près identique par toutes les usines métallurgiques. Les marques seules les différencient ét en rendent la recherche un peu délicate. Aussi, croyons-nous rendre service aux lecteurs de cette revue en leur donnant un tableau de correspondance des différentes marques d'aciers français pour les nuances dont nous venons de parler. Tel est l'abjet de notre tableau II.

Il ne faudrait pas croire pourtant que les quatorze nuances d'acier que nous venons de signaler sont les seules qui sortent de nos aciéries. Un simple coup d'œil sur un catalogue permettra de se rendre compte gu'il cxiste bien des intermédiaires dont les qualités répondent à tous les desiderata ; mais ce sont les quatorze nuances fondamentales auxquelles un constructeur pourra loujours se xeporter et parmi lesquelles il trouvera le plus souvent les caracléristiques dont il a besoin.

Ces caracléristiques, que nous avons donnérs (Tableau I), se rapporfent généralement à deux états nettement distinctș : l'un recuit, l'autre soit uniquement trempé, soit trempé et revenu, la trempe cllc-mème étant une trempe à l'eau, à l'huile ou à l'air, suivant la nuance de l'acier. Les chiffres du tableau sont assez éloquents par eux-mêmes pour montrer l'influcnce de ces traitements sur les caractéristiques; mais on peut se demander pourquoi on n'indique que deux traitements et si Jeur choix a été réellement bien judicieux

C'cst pour répondré à celte préoccupation que nous avons Átabli les courbes de la figure $x$, qui indiquent les variations des caractéristiçurs des principaux aciers éludiés lorsque,

(1) Les Alpes Economiques, mars ig2o. après trempe, on lcur fait subir un revenu à diverses tompératures.

Quant ì la température et au mode de trempe adoptés pour chaque acier, ce sont ceux qui ont été indiqués au tableau I. Tous ces traitements s'entendent sur pièces de petiles dimensions : ronds de $\mathrm{r} / \mathrm{a} \quad \mathbf{a}, 6$ millimètres de diamètre. Pour les aciers ayant $u n$ peu de tendance à la
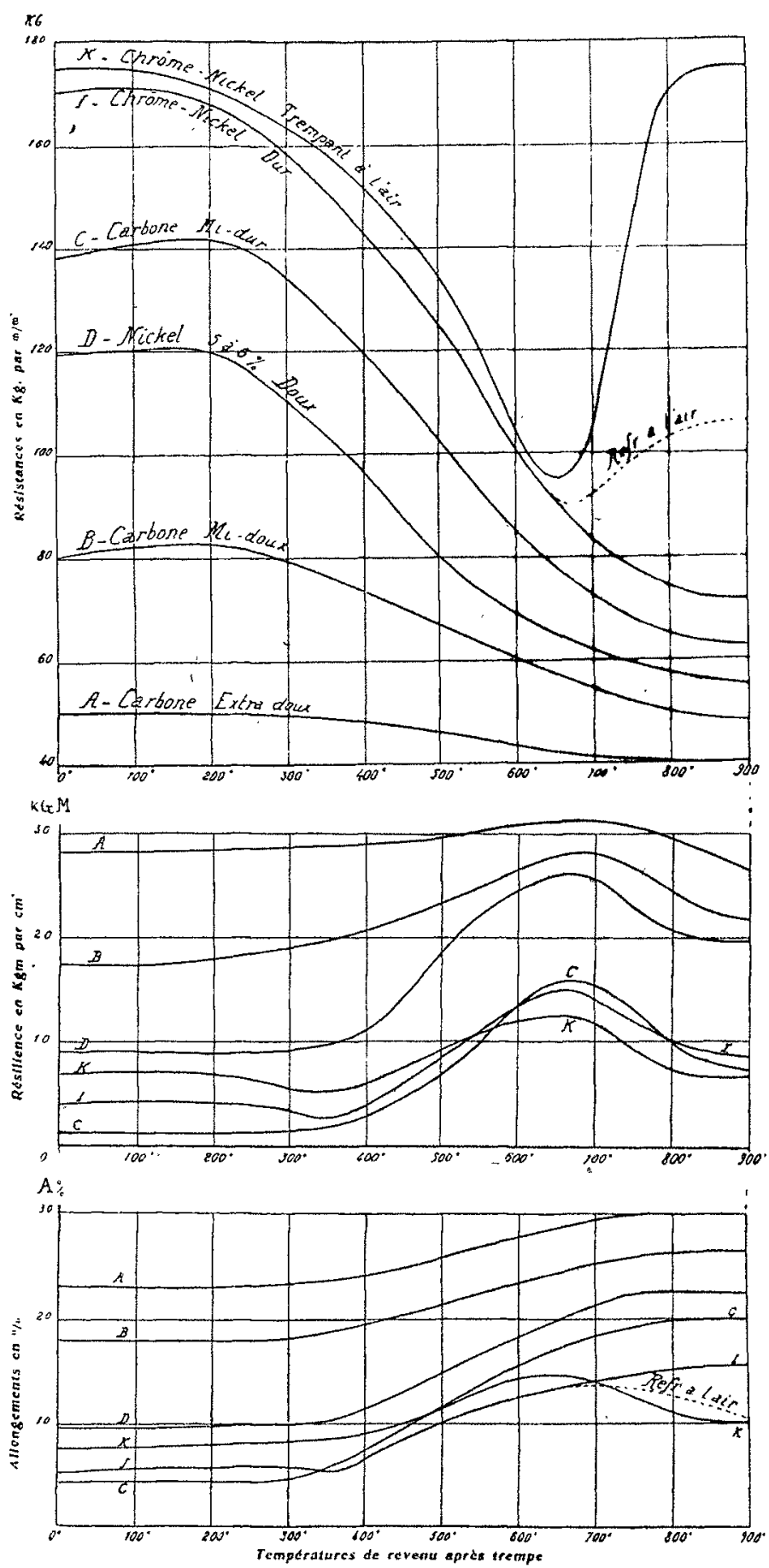

Fig. 1.- - Variation des caractéristiques mécaniques des principaux aciers de construction en fonction de la température de revenu.

trempe, le refroidissement après revenu a toujours été relativement lent (refroidissement dans la chaux à $30^{\circ}$ ), saul pour l'acier $\mathrm{K}$ (auto-trempant), et pour les courbes pointillées de l'acier I (Chrome-Nickel dur), ani correspondent à un refroidissement à l'air après revenu.

Outre leur intérêt documentaire, nous allons lirer de ces courbes, les bases d'une classification pour les aciers envisagés. 


\section{TABleau I}

\section{Compositions et Caractéristiques des principaux Aciers de Construction}

Etabli par la Chambre syndicale des Construcleurs d'Automobiles

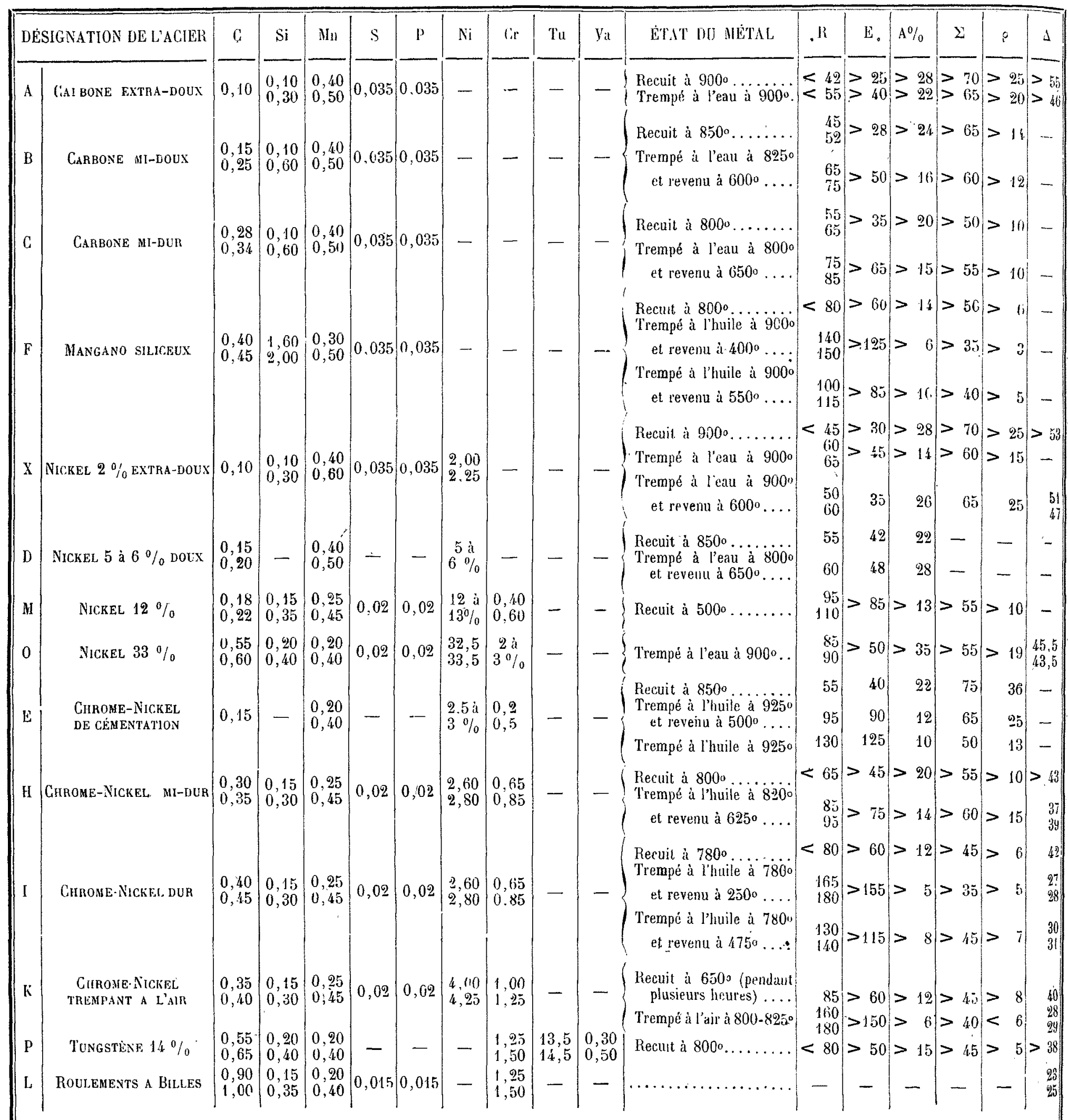

Les carnctíristiques sont obtenues avee l"éprouvette $13.8 \times$ Joo.

La striction $\Sigma$ par la formule $\frac{\mathbf{S}-\mathbf{S}}{\mathbf{S}} \times \mathbf{1 0 0}$

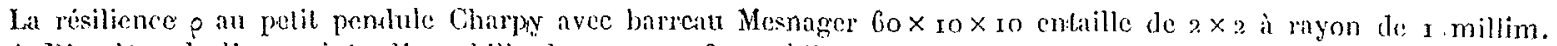

$\Delta$ Diamilre de l'empreinte d'une bille de ro, sous 3.000 kilogs.

Le carbone est dosé par combustion sìche et absorption par de la chatux solke contenue dins un tube laré.

Les compositions chimiques sont dounćes à tite d'indication et correspondent approximativement aux prineipales marques d'acier. 


\section{TaBleau II}

Correspondance approximative des différentes marques d'aciers de construction

\begin{tabular}{|c|c|c|c|c|c|c|c|c|c|}
\hline & & $\begin{array}{c}\text { Compagnie } \\
\text { des Forges de } \\
\text { Chatillon } \\
\text { Commentry } \\
\text { et } \\
\text { Neuves Haisons } \\
\text { (Montluçon) }\end{array}$ & $\begin{array}{l}\text { Compagnie } \\
\text { des Forges el } \\
\text { Aciéries } \\
\text { électriques } \\
\text { Paul GIRoD } \\
\text { (Ugine) }\end{array}$ & $\begin{array}{c}\text { Compagnie } \\
\text { des Forges et } \\
\text { Aciéries } \\
\text { de la Nlarine } \\
\text { et d'Ilomécourt } \\
\text { (St-Chamond) }\end{array}$ & $\begin{array}{c}\text { Jacob HoLT\%ER } \\
\text { el Cio } \\
\text { (Unicux) }\end{array}$ & $\begin{array}{c}\text { Scineider } \\
\text { et Cir } \\
\text { (Le Creusot) }\end{array}$ & $\begin{array}{c}\text { Société } \\
\text { Inonyme de } \\
\text { Conmentry- } \\
\text { rourchambaud } \\
\text { et } \\
\text { Decazeville } \\
\text { (Imphy) }\end{array}$ & $\begin{array}{c}\text { Socirle } \\
\text { Anonyme des } \\
\text { Hauts Fourneaux } \\
\text { Forges et } \\
\text { Aciéries du } \\
\text { Saut-du-Turn }\end{array}$ & $\begin{array}{l}\text { Société } \\
\text { Anonyme des } \\
\text { Forges } \\
\text { at Acièlies de } \\
\text { Fimilyy }\end{array}$ \\
\hline A & CARBONE EXTMA- IIOUX & $\begin{array}{c}\text { Soldat no } 8 \mathrm{et} \\
\mathrm{B} \mathrm{FM}\end{array}$ & f:0 & $\mid \begin{array}{cc}\begin{array}{lll}0 & 9 \\
\text { Pxtra-dous } & \text { et } \\
C & \Lambda & 0\end{array}\end{array}$ & & $A M \|$ et.AMC & $\begin{array}{lll}N^{0} & 10 & \mathrm{FF} \\
\mathrm{BC} C \mathrm{H} & \text { et }\end{array}$ & $\begin{array}{l}\text { Extra-doux et } \\
\text { "Perméa " }\end{array}$ & $\begin{array}{l}\text { No } 7 \text { extra- } \\
\text { doux el C'l }\end{array}$ \\
\hline$B$ & Carbone mi-nolix & Suldat 1107 & C $2-C 3$ & No 6 doux & & $\triangle \mathrm{M} 8, \wedge \mathrm{M} 9$ & $\mathrm{X}^{0} 8 \mathrm{DO}$ & Demi-doux & $\begin{array}{c}N^{0} 6 \text { doux } \\
\text { No } 5 \text { mi-doux }\end{array}$ \\
\hline $\mathrm{C}$ & Carbone mi-dur & Soldat no 6 & C 4. & $\begin{array}{l}N^{0} 3 \text { dur } \\
\mathrm{N}^{0} 4 \mathrm{dur}\end{array}$ & & $\Lambda \geqq 6$ & $\mathrm{~N}^{0} 5 \mathrm{l} \mathrm{l} \mathrm{T}$ & $\begin{array}{c}\text { Dur-tenace } \\
\text { Dur }\end{array}$ & $\mathrm{N}^{0} 4$ demi-dur \\
\hline $\mathrm{F}$ & MaYgano SILICEUX & S E & MS et MSK & S S & $\mathrm{Rs}$ & $M L$ & NOS et MWs & $\mathrm{MNS}$ & $\operatorname{CiRN}$ \\
\hline$x$ & NiCKEL $2 \%$ EXTRA- DOUX & “Incassalle & $\mathrm{N} 2 \mathrm{C}$ & $2 \%$ de Ni doux & & $\mathrm{DNC}$ & 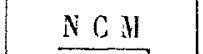 & “Perméa $n^{0} 2 "$ & CTN 2 \\
\hline D & Nickel 5 à $6 \%$ voux & $S P n^{0} 3$ & N $5 \mathrm{C}$ & $\begin{array}{l}5 \text { à } 6 \% \\
\mathrm{Ni} \text { doux }\end{array}$ & N 6 & 5 DC et 5 D 6 & N $5 C M$ et ND & $\frac{\text { "Permea }{ }^{0} 5 n}{\text { et }} \frac{1 N 5}{N 5}$ & $\cos 6$ \\
\hline$M$ & NICKEL $12 \%$ & & & $10 \mathrm{a} 12 \%$ de $\mathrm{Ni}$ & $N 27$ & $12 \mathrm{D}$ & & & \\
\hline 0 & Nickel, $33 \%$ & & & $30 \%$ de $\mathrm{Ni}$ & N 32 & $33 \mathrm{bDF}$ & NAS Chromé & & N. 30 \\
\hline E & CIROME-NICKEI DE CÉMENTATION & & G1. & & $\mathrm{N} 2+$ & DFC & $\underline{N C R}$ & & C'T N V \\
\hline II & Chrome-NICKEL MI-DUR & CR-NI n 2 & $\mathrm{~K} \mathrm{~N}$ & $\mathrm{CN} 4$ & GN 5 & D F $5 \mathrm{~S}$ & NG 3 il 1 & 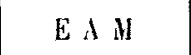 & NC 3 \\
\hline 1 & GIIRONE-NICKEL DUR & GR-NI $n^{0} 1$ & $\mathrm{KNH}$ & & C N $5 \mathrm{D}$ & DF $3 \mathrm{~S}$ & $\mathrm{NC} 3 \mathrm{H} 2$ & E $A D$ & NC: 2 \\
\hline $\mathrm{K}$ & CIROME-NICKEL TREMPANT A L'AIR & $\mathrm{SP}+$ & $\mathrm{K} N \mathrm{~A}$ & V I R & $\mathrm{C} \times 6$ & $4 \mathrm{DF} 2$ & $\mathrm{~B} \mathrm{Y}$ & $\mathrm{FR} \|^{\circ} 2$ & VDL et VIJLD \\
\hline l & TUNCSTÈnE $14 \%$ & $\begin{array}{l}\text { Rapide } S J \\
n^{0} 2\end{array}$ & Mars & Pliénix D 2 & Express E & S 2 & Extra-rapide & Le Précieux & Eclair \\
\hline$L$ & Rouleanents a BrLees & $\mathrm{RB}$ & K S & $\begin{array}{l}\text { Acier pour } \\
\text { roulements }\end{array}$ & Spécial $\mathbf{B}$ & F R & $\mathrm{T} \in \mathrm{i}$ et $\mathrm{TC} 2$ & $\mathrm{H} \mathrm{B}$ & $\mathrm{KOB}$ \\
\hline
\end{tabular}

\section{I. - Série des Aciers $\left(\begin{array}{l}\text { A - Carbone (extra-doux). } \\ \text { B - Carbone (mi-doux). }\end{array}\right.$ au Carbone.}

les acićries fabriquent jusqu’à dix ou douze nuances d'aciers au carbone, allant depuis une résistance de 30 à 35 kilos jus qừà roo kilos ; mais les trois nuances envisagées dans le tableau, $\mathrm{A}, \mathrm{B}$ et $\mathrm{C}$, sont bien effectivement les plus employécs pour la construction. Vu leur importance, nous avons donné (figure $\mathrm{r}$ ), les courbes complètes correspondant à ces trois aciers. Un simple examen de ces courbes permettra de se rendre compte des propriélés respectives de ces trois muances. Elles sont, du reste, trop connues pour qu'il $\mathrm{y}$ ait lieu d'insister.

$$
\begin{aligned}
& \text { II. - Série des Aciers } \\
& \text { spéciaux. }
\end{aligned}\left\{\begin{array}{l}
X-\text { Nickel } 2 \% \text { (cxtra-doux). } \\
D \text { - Nickel } 5 \text { à } 6 \% \text { (doux). } \\
\text { II - Chrome-Nickel (mi-dur). } \\
I \text { - Chrome-Nickel (dur). }
\end{array}\right.
$$

Nous trouvons, dans celte sćric, une gamme complète à

durcté croissante des aciers spéciaux pouvant être employés a la place des aciers au carbono toutes les fois où l'on a besoin de caractéristiques (limite élastique, allongements, résilience, aptitude à la trempe en profondeur) supérieures à celles que l'on obtient avec les aciers au carbone de même durcté, et que l'on n'est pas arrêté par lo prix de revient plus élevé. En ontre, la nuance dure des aciers au carbone ne peut guère, à causc de sa fragilité, être ulilisée comme acier de construction; elle est presque exclusivement réservée aux pièces d'outillage el ne figure pas parmi les aciers de construction. Il n'en est pas de même des aciers spéciaux.

Nous citerons, à titre d'exemple, quelques-uns des emplois de ces différents aciers :

Acier X. - A l'état recuit : spécial pour les emboutis i froid ; à l'étal trempé : diverses pièces d'autos el de machines ; arbres, engrenages, bielles, crochets et tendeurs d'altelage.

Acier D. - La dureté de cet acier pouvant être déjà fortement augmentéc par trempe el recuit, sans qu'il 
devienne fragile, son emploi est recommandé pour les pièces devant résister à de grands efforts, nolamment à des vibrations ou ì des chocs : arbres à cames, bielles de motcurs rotatifs, essienx et trains d'atterrissage, tiges de martuanpilons, etc.

Cet acier, qui n'est pas très différent comme dureté de l'acier $\mathrm{C}$ au carbone, a des allongements et une résilience qui lui sont de beaucoup supérieurs comme on peut s'en rendre comple par l'examen des figures.

Acier II. - Cet acier, qui acquiert une très grande dureló par trempe el recuit, sans fragilité, convient spécialement pour toutes les applications exigeant des garantics particulières de sécurité; têls les vilebrequins de moteur's d'autemubiles el d'aviation.

Pour ne pas embrouiller la figure $r$, nous n'avons pas fait ligurer les courbes correspondant à cel acier; mais, bien quayant une dureté notablement supérieure à celle de C, ses allongements et sa résilience sont très supérieurs.

Acier I. - Cel acier, intermédiaire entre les acicrs à trempe normalc el les aciers auto-trempants (ou trempants à l'air), comme on peut s'en rendre compto par l'examen des courbes pleines et pointillées, correspondant respectivement au refroidissemient lent et au refroidissement à l'air" après revenu, a une très grande aptitude à prendre la trempe en profondeur. Par suite de sa grande dureté, il résistic fort bien à l'usure ef convient particulièrement bien pour les engrenag̀es non cémentés à grande résistance.

Notablement plus dur que l'acier $\mathrm{C}$ au carbone, cet acier n'en est pas moins, dans la plupart des cas, scnsiblement moins fragile.

III. - Aciers $\left\{\begin{array}{l}\mathrm{A}-\text { Carbone (extra-doux). } \\ \mathrm{X}-\text { Nickel } 2 \% \text { (extra doux). } \\ \mathrm{D}-\text { Nickel } 5 \text { à } 6 \% \text { (doux). } \\ \mathrm{E}-\text { Chrome-Nickel (de cémentalion). }\end{array}\right.$

Les aciers $A, X$ et $D$ s'emploient suivant les cas, cémentés ou non cémentés ; toutefois, la plupart des aciéries ont créé deux nuances très voisines, mais cependant différentes pour l'un et l'autre cas. L'acier de cémentation doit être, cn effet, à teneur en carbone aussi faible que possible; c'est ainsi que l'acier $D$ de cémentation ne devra pas contenir plus de o, 150 de carbone au grand maximum. Dans le tableau II; nous avons souligné les marques spéciales de cémentation.

L'obligation d'avoir une teneur en carbone très faible ne permet d'obtenir des nuances diverses parmi ces aciers de cémentation qu'en agissant sur les constituants spéciaux. L'acier de cémentation au carbone est donc unique (A). Les aciers $X, D$ et $E$ nous représentant la gamme des aciers extra-doux (X), mi-doux (D) et mi-durs (E) de cémentation sont donc forcément, pour les deux derniers an moins, des aciers spéciaux. Ceux dont nous parlons sont au nickel ou au nickel-chrome.

L'emploi des pièces cémentécs est trop connu pour qu'il soit nécessaire d'insister. Lés considérations sur la dureté respective et la composition de oes quatre aciers différents suffit pour fixcr le choix dans chaque cas particulier.

$$
\begin{gathered}
\text { IV. - Aciers trempant à l'air } \\
\text { (auto-trempants). }
\end{gathered} \quad \begin{gathered}
\mathrm{M}-\text { Nickel I } 2 \% . \\
\mathrm{K}-\text { Chrome-Nickel } \\
\text { trempant à l'air. }
\end{gathered}
$$

Une des caractéristiques les plus iutéressantes de ce genre d'acier est. que sa durcté, à l'élat trcmpé, est la même dans toute la profondeur d'une pièce, quelle que soit l'épaissemr. La trempe de ces aciers ne les déforme nullement.

L'acicr $M$ et l'acier $K$ s'emploient pour toutes les pièces qui, en raison de leur travail exceptionnel, exigent une rigidité spéciale telle que claveltes, boulons et écrous spéciaux, arbres, tiges de freins, elc.

On doil signaler, en outre, bicn 'que cela sorte un peu de notre sujel, la très grande résistance de l'acier $\mathrm{K}$ aux projectiles d'où son cmploi comme plaque de blindage, soit it l'étal naturol pour les blindages relativement minces contre les petits projectiles, soit à l'élat cémenté cl traité pour les blindages épais contre la grosse artillerie.

\section{V. - Acier à grand allongement et, très résistant à la corrosion. \\ $\mathrm{O}$ - Nickel 33.}

Le tableau I né renferme qu'un senl acier de cc gemre, l'acier 0 . Son inoxydabilité le désigne tout particulièrement pour les pièces devant travailler à l'humidité et ì la vapeur. Il résiste également bien aux chocs répétés el à la corrosion par les gaz porlés à haute température, c'est ce qui le fait employer pour cerlaines pièces de motcurs a explosion.

$$
\text { VI. - Aciers à emplois }\left\{\begin{array}{l}
\mathrm{F} \text { - Mangano-Siliceux. } \\
\mathrm{P} \text { - Tungstène } \mathrm{I} / 4 \% \text { \% } \% \text {. } \\
\text { speciaux. }
\end{array}\right.
$$

Acier F. - Nous atlirons l'attention sur la dénominatimi un peu défectueuse de ccl acier, appelé silico-maganeux par les uns, il est désigné par esprit d’imitation et de contradiction, égalemient par d'autres, sous le nom de manganosiliceux. Or, c'est tout simplement un acier au silicium rini est spécialement utilisé pour la fabricatión des ressorts, en raison de la limite élastique élevée qu'il peut atteindre ì. l'étal trempé et recuit, sans que sa fragililé soil trop exagérée. Cet acier est également ulilisé pour les engrenages et pour les pièces travaillant à la flexion ct à la torsion commé les vilebrequins.

Loin de favoriser la trempe, le silicium a plutôt tendance à le rendre plus difficile. On ne doit donc pas craindre de tremper énergiquement cet acier. On préconise même son: vent une trempe beaucoup plus énergique que celle indiquée au tableau I, c'est-à-dire trempie à l'cau à des températures pouvant aller jusqu'à rooo". Le recuit est ensuite proportionné à la dureté finale que l'on désire : 200 à $300^{\circ}$ pour les ressorts ; $400^{\circ}$ pour les éngrenáges, $650^{\circ}$ pour les: vilebrequin's.

Il n'est pas sans intérêt de signaler que cet acier, utilisé cependant depuis fort longtemps, a recu, pendant la guerre, une application nouvelle qui lui a donné un regain d'actualité. Il s'agil de son emploi pour les cylindres de moleurș d'aviation. On a reconnu, en effet, à l'usage, que les cylindres ainsi constitués résistaient micux aux lompéralurcs élevées auxquelles ils travaillent, que les acicrs au carbone de dureté correspondanle.

Acicr P. - Comme acicr de construction, on utilise h propriété de cel acier (crui n'est autre qu'un acier à oulil. rapide) de conserver à température élevée des caractéristiques mécaniques beaucoup plus élcrées que les autres aciers. Or' dans les moteurs à explosion, très poussé, comme c'cst le cas des moteurs d'avialion, les soupapes d'échappement peuvent atteindre jusqu'à $800^{\circ}$. Cet acier est donc tout indiqué.

Acier L. - Le roulement à billes constitue une fabrication bien spéciale nécessilant un acicr trempant fortement. Cet acier au chrome est universellement adopté pour cet usage.

Nous allons maintenant comme conclusion résumer dans. lc Lableau III les principaux cmplois des différents aciers, que nous venons d'envisager. 
Tableau III

\section{Emplois des principaux Aciers de construction}

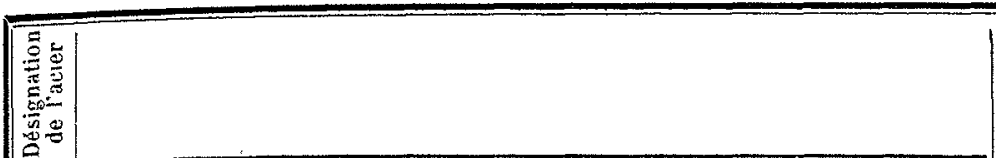

Etat non cémenté. - Acier pour tréflage et étirage. - Conduite d'eau rivée (les conduites soudées nécessitent une nuance particulière ayant sensiblement la même composition chimique, mais élaborée spécialement). - Jantes d'automobiles. - Maillon pour chaînes de Galle. - Rivets. - Tôkes et pièces cmbouties à frojd, ou reppoussécs au marteau.

Etat cémenté et traité. - Cet acicr est spécialement fabriqué en vue de la cémentation pour toutes les pièces destinées à travailler au frottement. - Arbre de trains baladenrs. - Arbres, bielles et axes divers. - Axes de pistons. - Cames. - Cônes. - Comronnes de différenticls. - Cuvettes. - Engrenages. Mouvernents de pédaliers. - Pignons. - Poussoirs. - Pièces diverses pour automobiles et bicyclettes.

Adores de transmission. - Albres divers. - Barres d'excentriques. - Bielles diverses. - Boulons ordinaires. - Cadres de tiroirs. - Colliers d'excentriques. - Crosses de pistons. - Essienx droits de lacomolive. - Fssicux de wagons et de tramways. - Ferrures diverses. - Longerons. - Pistons. - Plaques de garde. - Poinçons à chand. - Profilés el toles pour châssis d'automobile, pour ponts el charpentes. - Tiges de pistons. - Tôles pour coques. - Vis. - Pièces liverses pour machines.

Cet acior est tròs employé pour la construction méonnique grénérale et porr la construtction automobile. - Arbres. - Axes. Moyeux. - Essicux. - Bielles. - Pièces de châssis. - Carters. - Claveltes. - Crochets d'attelage. - Cylindres ac moteurs. Disques de turbinos. - Etampes à chaud. - Ferrures diverses. Fusćes d'cssiemx. - Glissières. - Outills agricoles : pelles, bèchès, socs ct versoirs de charrues, cte. - Ressorts. - Tendeurs d'attelage. - Pièces d'arnms. - Boulows, vis, ćcrous.

Acicl supéricur pour lames de ressorts at ressorts de touter sortes. - Engremnges, - Pièces travaillant à la flexion ou à la torsion comme les arbes-vilcbrequins. - Cylindres de moteurs d'aviation.

$\mathbf{x}$

Elat non rémenté. - Pièces d'altelage de chemin de fer. Essienx d'alutomobile. - Fusées, bonlons, écrous. - Rivets pour conshruction supéricure.- Tiges de pilons.- Leviers de dircction.

Etat cémenté et traité. - L'emploi dans les mêmes conditions que l'acier A cémenté, inais pour des fabrications plus soignées, car il donne une sécurité plus grande que ce dernier.

D

Etat nòn cémenté. - Ailettes de turbines à vapeur. - Soupapes de moteurs. - Prôfilés pour châssis rigides. - Tiges de pilons.

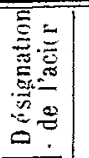

- Tôles pour châssis rigides. - Tubes spéciaux, pour trains d'attérissage par exemple.

Etat cémenté et traité. - Possìde une plus haute rósistance apres trempe que les aciers $A$ et $X$. - S'emploie dono dans les mèmes cas où on a des pièces faliguant beaucoup et devant résister à l'usure par frottement. - Arbres de vilebrequins. - Axes de pistons. - Bielles diverses. - Champignons de soupapes. Engrenages. - Essicux AV el AR d'automobile. - Fusúes d'es. sicux. - Pignions.

Clavetes, boulons et ćcrous speciaux. - Arbres. - Profilés pour construction très résistante. - Tiges de froins de canons.

Acier inoxydable"spécialement désigné pour pieces devant travailler à l'humidité el à lá vapenr et la corrosion par les gaz chauds. - Clapets, solupapes de moleurs. - Tiges de pompes.

Etat cémenté et traití. - Prósente après cómontation at traitement (trempe el revenu) non seldement whe tres grande durete superficielle ; mais la partis intericure elle-mème a une très grande résistanes et une limile élastique dovéc. - Axes de pistons. - Boulons spéciaux. - Champignons de soupapes. Engrenages. - Tubes spéciaux pour trains d'atterrissage, atc.

C'est le plus employé des aciers pour pieces do machines délicates. If joue un grand rôle dans la conslruction, automobile. l'ariation, les organes les plus essentiels des torpilleurs is grande vitesse, elc. - Arbres de dynamos, de lurbines. - Arbre vilebrequins. - Boulons speriaux. - Disques de lurbines. - Basieux droits pour locomotive, wagons, tramways. - Manivelles. - Tiges de pistons. - Tôles pour usages spécinux, ek., elc.

Acier extrêmement dur après traitement, (trempe à l'huile ayoe revenu à $250^{\circ}$ ou mème sims vevenu), résiste particulièrement bien à l'usure par frottement, Il est lonc l'acier idéal pour les engrenages non cémentés à hautc résistanco.

$\mathbf{K}$

Cet acier donnis par simple trompe is laij des résultats analo. gues à ceux du précédent trompé ì llhuile. II convient done it des emplois du mern grenre et 11 a pas à eraindre les déformations de trempe. - Fingrenages. - Arbres à côtre. - Matrices. - Tôles de masque.

$\mathbf{P}$

Acier spécial pour pièces devant travailler ì des températures élevées comme soupapes d'échappement des moteurs d'aviation.

I.

Acier ayant une très grande rósistance ì l'usuxe et à l'écrasement. - S'emploie spécialement pour tubes, barres, galets pour roulements à billes. - Billes. - Grains de butée. - Grapau. dines, etc.
Nora. - Les chiffres de résilience indiqués dans le tableau de la Chambre syndicale des Constructeurs d'automobiles et ceux , rue nous donnons en courbes dans notre figure, ont été obtenus en cassant au mouton pendule Charpy dê 30 kilogrammètres des barreaux du type Mesnager de $60 \times 10 \times 10$, entaille de $2 \times 2$ à rayón de $1 \mathrm{~m} / \mathrm{m}$ (voir fig. 2 ).

On sait combien les essais dits essais de fragilité sont quelque chose d'artificiel. Le chiffre de résilience (nombre de kilogrammètres nécessaires pour obtenir par choc la rupturd de la sèction nón entaillée d'un barreau ; section rapportée à $\mathrm{r} \mathrm{cm}^{2}$ ), ne dépend pas seulement, comme les autres constantes d'essais, de la nature du métal ; il dépend aussi, dans de fortes proportions, de l'appareil 'd'essai el surtout des dimensions. du barreau et de la forme de son enlaille. En outre, la force vive absorbéc par la rupture du barreau caractérise non seulement son aptitude à la rupture pax fragilité, mais encore bien souvent, son aptitude ì la déformation, car les barreaux entaillés d'acier doux se dóforment notablement pendant l'essai de choc. Les ruptures accidentelles dites ruptures par fragilité, auxquelles ces mêmes aciers sont parfois sujets lorsçu'ils sont utilisés dans la pratique, accusent, au contraire, des déformations très faibles.

Cet essai, tel qu'il est actuellement pratiqué, est done très imparfait, tout le monde est d'accord sur ce point ; mais il donne cependant des renseignements intércssants qui, faute de micux, ne doivent pas être nég]igés. Il faut seulement se bien persuader que les résultats de l'essai ne signi- 
ficnt rien en valcur absolue, et qu'ils ne peuvent donner des indications susceptibles de guider, même à titre comparatif', que s'ils sont obtenus dans des conditions en tous points jdentiques.

Ces considérations el d'autres d'un ordre peut-être un peu moins élevé, avaient rendu les industrièls très circonspects rclativement à ce genre d'essai. Toutefois, à la suite d'unc sorte d'entente tacitc, les métallurgistes avaient fini par accepter l'essai de fragilité tel qu'il est défini dans le tableau I : barreau Mesnager (fig. 2) cassć au pendule Charpy.

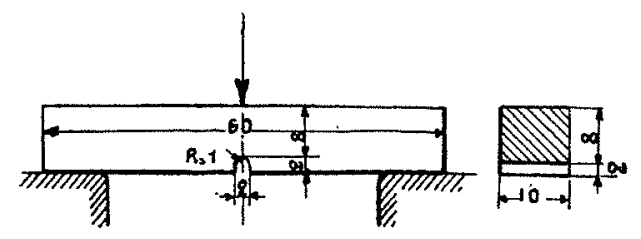

Fig. 2. - Barreau Meennger.

C'est ainsi que nos courbes (fig. I) onl été obtenues. L'obtention de telles courbes, on le comprendra facilement, nécessilent un nombre considérable d’essais très coûtcux. $\mathrm{Or}_{\mathrm{r}}$, la documentation très péniblement établic jusqu'ici va devenir, à peu près inutilisable, car la Commission Permanente de standarisation a rendu officiel, pour cel essai, un type de barrean différent de celıi qui avait élé accepté officieusement jusqu'ici.

Ce nouveau type dont on trouvera te croquis (figure 3) est un compromis entre le barreau Mesnager el le barreau Charpy.

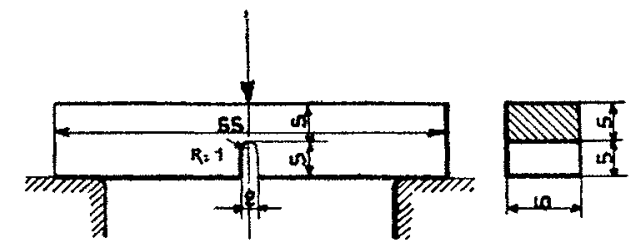

Fig. 3. - Bitreau de la Commission Permanente de standardisation.

Nous n'ayons pas l'intention de criliquer, ici, la décision do la Conmission Permanente de Standardisation qui a adopté ce nouveau barreau sur la proposition des savants les plus compétents el les micux cualifiés, qui avaient été spécialement chargés d'étudier cette question ; mais nous tenons à souligner la répercussion assez considérable que va avoir celle décision et les travaux importants qui devront être faits pour mettre la documentation sur la fragilité en concordance avec le nouveau type d'épreuve. On ne peut espérer, en effet, passer des essais anciens aux nouveaux, en appliquant un simple coefficient. Des-éludes récentes de M. Guillel (1) d'une part et de M. Legrand ( $\left.{ }^{1}\right)$ d'aulre part, montrent que, pour les aciers doux, la résilience observée avec le nouveau barreau ast égale environ aux $2 / 3$ de colle que donnait lc barreau Mesnager ; quant aux aciers durs, il y a à peu près identité.

Ces quelçués réflexions suffisent à montrer la complexité du problème de la fragilité des métaux el l'inlérêt que présentent les études qui tendent à échaircir un peu celte queslion forl délicate.

\section{P. Dejean,}

Directeur du Laboraloire des Essais Mécaniques el Mélallurgiques de l'Inslitut Polylcchnique de l'Université de Grenoble.

(I) Revue de Métallurgie, avril igar.

IFGISIATION

LE RACHAT

CONCESSIONS DE CHUTES D'EAU

\begin{abstract}
On nous a demandé à plusieurs reprises de préciser les conditions dans lesquetles doil, ou peul élre opéré le rachat d'une concession hydraulique, clonnée d'apris la loi du 16 oclobre 1919 el en conformeté da cahier lype approuvé par décret du 5 septembre 1920) (woir le lexte à mon Manuel sur la Législation Nouvelle des Chutes d'Eau, page 219).

Nous reconnaissons qu'il $y$ a sur ce point cerlaines obscurités à éctaircir et nous avons l'intention dats le présent arlicle de nous $y$ employer de notre micux.
\end{abstract}

\section{I}

D’une façon générale, l'autorité administrative n'adum plus aujourd hui qu'elle puisse donner une concession, pour un temps assez long, sans préroir à son profit te droit absolu de reprendre ce qu'elle a donné, ou... pour parter plus exactemenl, de prendre pour la première el deruière fois, ce que le concessionnaire a śtabli. lin effet, un antatcur de précisjon dans le stylé, se demande pour qucl motif on parle du "rachat " des concessions : pour "racheter", il faut avoir vendu une première fois. Je dis couramment, par excmple, qu'après avoir aliéné mon immeuble, je l'aj "racheté " parce qu'à la suite d'un regret, j'ai voulu m'en rendre à nouveau propriétaire. Mon acquéremr en avait fait "l'achat ", je procède à une scconde opération "d'achat" el, bien que les rôles aient changé puisque je suis acheleur de ce cque j'ai vendu, on ponul dire qu'il y a "rachal "parce qu'en réalité, il y a un deuxième " achal ". Mais quand l'autorité supérieure m'a donné, dans un cahier des charges approuvé par décret, un titre nu grâce auq̣ucl j'ai cu le droit de faire des travaux pour mettre en valcur lia puis: sance de l'eau, et quand elle me demande en cours de concession d'abandonner la jouissance des travaux que j'ii faits, de mes propreś deniers, on cherche comment il peut être parlé de " rachat ", puisq̣ue ces ouvrages 'ne m'ont jamais été vendus, ni achotés.

Quoi cru'il en soil, le terme est bien ancré dans nos usagyes et il y restera d'autant plus sûrement qu'il est faux.

La première loi qui a affirmé l'intention du Gouvernemenl de ne plus donner ou laisser donner de concession, de pea d'mporlance, sans permcltre à l'aulorité concédante do la rompre, est la loi du onze juin $r 880 \quad\left({ }^{1}\right)$ sur les (hremins de fer d'intérêt Jocal, où nous lisons ce qui suit: " à lonte époque, une voic ferréc peut être distrailé "du domaine public, déparlemental ou communal et " classéc par une loi dans le domainr de l'Etal. Dans co " cas, l'Etat csl substitué aux droits ct obligations du dépar" " lement el de la commune à l'égard des entrepreneurs d " des concessionnaires tels que ces droits et obligations résul" tenl des conventions légalement autorisées. En cas d'évic" tion du concessionnaire, si.ces droits ne sont pas réglés, " soit par un accord préalable, soil par le cahier des char-

(3) La loi de 1880 a lạissé la place à unc Joi nouvelle du $x^{3}$ juill. 'tgrt? dont te texte se trouve à la Revue des Concessions, Volume $N^{\text {to }}{ }^{3}$ annéc I9r3. 